\title{
Central role of obesity in endothelial cell dysfunction and cardiovascular risk
}

José Carlos de Lima-Júnior ${ }^{*}$

(iD) Alexandre Moura-Assis ${ }^{2,3^{*}}$

(iD) Riobaldo M. Cintra

(D) Thiago Quinaglia ${ }^{1}$

(iD) Lício A. Velloso 2,3

iD Andrei C. Sposito ${ }^{1}$

1. Laboratory of Vascular Biology and Atherosclerosis, Department of Internal Medicine, State University of Campinas (Unicamp), Campinas, SP, Brasil 2. Laboratory of Cell Signaling, Department of Internal Medicine, State University of Campinas (Unicamp), Campinas, SP, Brasil 3. Center for Research on Obesity and Comorbidities, OCRC, State University of Campinas (Unicamp), Campinas, SP, Brasil ${ }^{*} \mathrm{JCL}$ and $\mathrm{AMA}$ contributed equally to this review.

http://dx.doi.org/10.1590/1806-9282.65.1.87

\section{SUMMARY}

Atherosclerosis is the leading cause of mortality in the contemporary world. The critical role of the endothelial cells (EC) in vascular homeostasis, the metabolic changes that take place when the cell is activated, and the elements involved in these processes have been widely explored over the past years. Obesity and its impact, promoting a rise in blood levels of free fatty acids (FAs) are often associated with atherosclerosis and cardiovascular mortality. However, the mechanisms that promote cardiovascular structural changes and adaptive changes in the ECs, particularly in the context of obesity, are little known. Here, we reviewed studies that assessed the metabolic adaptations of healthy and dysfunctional ECs during exposure to FAs, as well as the epidemiological perspectives of cardiovascular structural changes in obesity. Finally, we explored the role of new agents - sphingolipids, dietary unsaturated fatty acids and sodium-glucose cotransporter-2 inhibitors (iSGLT2) - in atherosclerosis and their relationship with obesity.

KEYWORDS: Obesity. Risk factors. Atherosclerosis. Endothelium.

\section{INTRODUCTION}

Atherosclerosis is the leading cause of mortality worldwide and is associated with obesity. This disease has a physiopathological component key to the dysfunction of ECs, the cells that make up the luminal surface of blood vessels'. The activation of the endothelial cell by different biochemical or biomechanical stimuli results in an inflammatory phenotype, with loss of the homeostatic function as a micro-barrier, expression of adhesion molecules and prothrombotic molecules on its surface, as well as the generation of reactive oxygen species (ROS), which results in the progression of the atherosclerotic injury in the vessel wall ${ }^{2}$. This review focused on epidemiological aspects involving the association between obesity and atherosclerosis and how the basic understanding of metabolism and the ECs signaling during their exposure to FAs or other atherogenic components of the obese environment have contributed in providing 
insights and identifying potential therapeutic targets for the obesity/atherosclerosis binomial. In the final part of the review, we will discuss the roles of molecules or other substances identified more recently or fewer studies, sphingolipids, dietary unsaturated fatty acids and sodium-glucose cotransporter-2 inhibitors (iSGLT2), and their potential involvement in the physiopathology or therapy of the cardiovascular changes that accompany obesity.

\section{OBESITY AND RISK OF CARDIOVASCULAR DISEASE}

The rapid increase in the prevalence of obesity in Brasil and in the world over the last few decades was accompanied by a parallel increase in other comorbidities associated with obesity ${ }^{3}$. The epidemiological evidence establishes an association between obesity and mortality; however, there are controversies in the relationship between obesity and cardiovascular risk, and that seems to be associated with the pattern of body fat distribution and metabolic factors that involve insulin sensitivity, the profile of hormone secretion of the adipocytes and cardiorespiratory fitness ${ }^{4}$.

The heterogeneity of obesity and the concept of "obesity paradox," in which individuals with overweight and obesity present an improved cardiometabolic prognosis in comparison with eutrophic individuals, are fertile ground for an investigation of the mechanistic and physiological fundamentals of the diversity of effects of obesity on cardiovascular health ${ }^{5,6}$.

Although widely used in epidemiological studies and the clinical routine, the body mass index (BMI) is unable to distinguish areas of concentration of white adipose tissue. The local distribution of white adipose tissue and its impact on the cardiometabolic risk have been described since the 1940s, and the deposits of ectopic fat - deposits in visceral organs - and in the abdominal cavity are significantly correlated with cardiometabolic abnormalities ${ }^{7}$.

The deposits of visceral fat are more resistant to the insulin action and release free FAs in a higher proportion in comparison to the subcutaneous adipose tissue. The excess of free FAs in the bloodstream is closely related with the onset of inflammation, notedly observed through the increase in the serum levels of C-reactive protein, interleukin-6, and tumor necrosis factor- $\alpha$ (TNF- $\alpha$ ) in peripheral tis- sues $^{4}$. Additionally, the excess of circulating FAs and their influx into the hepatic portal system triggers a higher production of VLDL by the liver. In this context, although many obese individuals present standard levels of low-density lipoproteins (LDL-c), most of the LDL-c produced are small and dense particles, classically more atherogenic ${ }^{8}$. In rodents, perivascular fat and the role of the resistance to the effect of insulin on vascular cells during atherosclerosis are well documented. These studies demonstrate that the resistance to the effect of insulin on vascular cells reduces the bioavailability of nitric oxide and favors the adhesion and infiltration of immune cells, compromising the vasodilation properties and favoring the endothelial dysfunction ${ }^{9,10}$.

Taken together, the mechanisms mentioned above constitute some of the pillars of the heterogeneity of obesity and the physiopathology of cardiovascular diseases associated with obesity. The following topics discuss these interfaces in greater detail.

\section{METABOLIC ADAPTATIONS IN THE ENDOTHELIAL CELL IN OBESITY - BIOCHEMICAL PERSPECTIVES}

In healthy conditions, the endothelium coordinates the formation of blood vessels and keeps the vascular homeostasis and its structure. In pathological conditions, however, the metabolic changes that take place in the ECs can promote dysfunction and be a trigger event for atherosclerosis'. ECs support different cellular processes through several metabolic pathways, which can have different signatures according to bad metabolic adaptations, of which the understanding might help us prevent injuries and identify new therapeutic targets ${ }^{11}$. Initially, we present the fundamental aspects of the endothelial metabolism of blood glucose and Fas, ${ }^{12}$, both the most critical energy-generating pathways in healthy ECs, before exploring which fundamental changes in these mechanisms are present in the endothelial cells of obese individuals.

Counter-intuitively, in ECs that are very close to an environment where there is direct exposure to high blood flow, the ATP generation dos not rely, primarily, on the oxidative phosphorylation (Oxphos). ECs have a low mitochondrial density, and their generation of adenosine triphosphate (ATP) occurs mostly through anaerobic glycolysis, a process that occurs at a similar rate to the the one in cancer cells, 
for example, in which the glycolytic flux also prevails in relation to the oxidation of FAs and blood glucose (> 200x). Thus, even though the ATP generation through anaerobic glycolysis is less efficient than Oxphos, in a cellular environment where there is abundant availability of glucose, glycolysis becomes a efficient option ${ }^{12,13}$. Besides, that non-oxidative cellular approach decreases the generation of reactive oxygen species associated with Oxphos, as well as reduces the use of oxygen, making it available, primarily, for perivascular cells. Furthermore, this process allows the ECs to use the lactate protectively, as a molecule for controlling the angiogenesis ${ }^{12,14}$. In parallel, an intermediary of the glycolytic flux feeds the pentose phosphate pathway, which results in the generation of glutathione (GSH), a molecule with anti-oxidant potential (ROS scavenging) $)^{1,15}$.

FAs represent another energetic pathway for the production of energy in the ECs (approximately 5\%); however, the primary use of the FAs that enter an EC is not generating ATP ${ }^{12}$. However, in the absence of glucose, the metabolic flow is diverted to the oxidation of the FAs (FAO) in a process regulated by the AMPK (adenosine monophosphate-activated protein kinase), a cellular metabolic sensor ${ }^{16}$. The plasma FAs enter the cell through passive diffusion or FAs translocase, which also acts as acyl-CoA synthetase, leading to the formation of fatty acyl-CoA. The fatty acyl-CoA is conjugated to carnitine through carnitine palmitoyltransferase 1A (CPTIA) before being transported to the mitochondria by the carnitine/acylcarnitine shuttle for $\beta$-oxidation. After entering the mitochondrial matrix, enzymes of the CPT2 family catalyze the formation of acylcarnitines back to the fatty acyl-CoAs, which enter the $\beta$-oxidation pathway ${ }^{17}$. Thus, one of the regulators that play an important role in FAO is the CPT1A, limiting the FA flux destined to $\beta$-oxidation. For example, the EC-specific gene silencing of CPT1A causes defects in cell proliferation (though it does not cause changes in other homeostatic elements, such as cell migration) and EC angiogenesis ${ }^{18,19}$. Such changes occur because, in ECs, FAO is crucial for the synthesis of deoxyribonucleotide, since the FAs are a source of carbon as critical as glucose and glutamine to the citric acid cycle in the ECs ${ }^{19}$. In this context, occurs the generation of aspartate and glutamate, precursors of nucleotides, which, in turn, are critical for the DNA (deoxyribonucleic acid) synthesis during cell proliferation'.

Obesity is associated with a high level of circulat- ing FAs, and that increase in supply is a metabolic challenge for the ECs since this process is also associated with the generation of reactive oxygen species and is deleterious to the cells (ROS) ${ }^{20}$. Most endothelial cells in adults are in quiescent form (QEC), unlike the cells in proliferation (PECs). The QECs are continuously exposed to an environment rich in oxygen in the peripheral blood, and, consequently, to the oxidative stress promoted by the ROs, capable of promoting endothelial dysfunction through the decoupling of the vasoprotective function of the endothelial nitric oxide synthase (eNOS) ${ }^{21}$. Studies have sought evidence of the mechanisms through which these cells protect themselves to keep the vascular homeostasis, which could lead to the identification of new therapeutic targets that promote vascular protection in a FA-rich environment, like obese individu$\mathrm{als}^{18}$. According to what has been stated above, PECs use the $\beta$-oxidation of FAs as a source of carbon for the synthesis of nucleotides during the proliferative stage of the angiogenesis ${ }^{19}$. Recently, Kalucka et al. ${ }^{22}$ demonstrated that QECs are not hypometabolic, as was believed.

On the contrary, QECs increase the FAO flux when they become quiescent. Surprisingly, they use the $\beta$-oxidation of FAs not for the synthesis of nucleotides, such as in PECs, but to keep the redox homeostasis through the regeneration of antioxidant molecules ${ }^{22}$. That understanding certainly contributes to the view that EC adopts protective mechanisms during stress situations and the use of FAs to maintain redox homeostasis is a key mechanism.

This view that there are adaptive mechanisms that protect against the excess of FAs has been recently described. In general, it is known that highly acute levels of free FAs induce a bad adaptation and a consequent endothelial dysfunction in vivo in humans due to a worsen vasodilation mediated by nitric oxide ${ }^{23}$, as well as promotes the activation of the inflammasome and the of inflammatory signaling cascade controlled by the factor nuclear kappa B (NF$\mathrm{kB})^{24}$. Similarly, obesity/insulin resistance also promotes in vivo endothelial dysfunction in humans ${ }^{25}$. In the same way, FAs promote apoptosis through a mechanism dependent on the generation of ROS using the $\mathrm{NAD}(\mathrm{P}) \mathrm{H}$ oxidase ${ }^{26}$, as well as the membrane saturation of the endoplasmic reticulum and the consequent endoplasmic reticulum stress, which also activates inflammatory pathways ${ }^{27}$.

On the contrary, increasing FAO through the in- 
duction of the CPT1A upregulation or the promotion of other metabolic sensors upregulation, such as the peroxisome-proliferator-activator-receptor (PPAR), is capable of reducing endothelial dysfunction and the EC apoptosis, therefore, being a possible therapeutic target for endothelial protection in situations in which there is an increased supply of FAs in the plasma $^{24,28}$. Besides, bariatric surgery can reduce markers of systemic inflammation and endothelial activation $^{29}$ significantly. This improvement in the endothelial dysfunction might be associated to better management of fatty acids by lean tissue after the surgery since there is a decrease of the systemic lipolysis during the intravenous overload of lipids, as well as better disposal of triglycerides and production of acylcarnitine after the bariatric surgery ${ }^{30}$.

\section{CARDIOVASCULAR STRUCTURAL ADJUSTMENTS IN OBESITY}

Obesity is a well-known risk factor; however, part of its effect on the cardiovascular structure is caused by the concomitance of other risk factors. The isolated expression of the excess of white fat, thus, would happen only in so-called metabolic-healthy obese individuals. The analysis of cohorts featuring individuals with these characteristics would allow us to understand which cardiovascular phenotype is related to obesity. The largest cohort, so far, in number of patients $(n=3,500,000)$, suggests that this action leads to an adjusted risk of heart failure two times greater (Risk ratio: 1.96; confidence interval of $95 \%$ : 1.86-2.06) and 50\% higher chance of coronary artery disease (Risk ratio: 1.49; confidence interval of 95\%: 1.45-1.54).0.46-1.81). This same study revealed a lower risk, but not insignificant, of cerebrovascular disease (Risk ratio: 1.07; confidence interval of 95\%: 1.04-1.11) also linked to obesity. The average follow-up time for these outcomes to occur was $5.4^{31}$. Another meta-analysis confirms these findings in different populations that differ only on whether or not there is an increase in the overall mortality of these patients in comparison with the general population $^{32,34}$.

These adverse cardiovascular outcomes are mediated by structural changes that precede them. The most important of these outcomes seems to be heart failure. The association of obesity with diastolic dysfunction and left ventricular hypertrophy is well established ${ }^{35,36}$, and both factors precede this outcome.
The increase in mass and the deficit of left ventricle relaxation seem to be related with an increase in the total peripheral vascular resistance determined by a chronic increase of blood volume, already suggested by the Framingham study ${ }^{37}$. Also associated with heart failure, the left ventricular systolic dysfunction, as the diastolic, is more frequent in obese individuals. There is a clear linear correlation between $\mathrm{BMI}$ and the ejection fraction of the left ventricle, as well as between BMI and the E/e' ratio (marker of the diastolic dysfunction), and these relationships follow the increase or reduction of body weight in a individual, thus showing the strength of the association between these parameters ${ }^{38}$. The reduction in the ejection fraction, however, seems to be slightly more connected to hyperglycemia and insulin resistance concomitant with the increase in adiposity. Hyperglycemia can increase the intra and extracellular glycation of proteins, increasing the stress oxidation, inflammation, and injuries to the myocardium, culminating in the rigidity and reduction of contractili$\mathrm{ty}^{39}$. However, there are also descriptions of ventricular systolic dysfunction in obese individuals with standard blood glucose and glycated hemoglobin, indicating there must be other mediating mechanisms.

In fact, obese individuals with no insulin resistance (Homa-IR $<2.5$ and blood glucose $<100 \mathrm{mg} / \mathrm{dl}$ ) or other components of metabolic syndrome present heart disease more often than non-obese patients. Subclinical atherosclerosis assessed by the coronary calcium score is two times more frequent in this group (Prevalence ratio: 2.26; confidence interval of 95\%: $1.48-3.43$ ), as shown by a cross-sectional study ${ }^{40}$. This same study, however, suggests that the diagnosis parameters for metabolic syndrome are linked to a higher calcium score, even though they are below the cutoff values, especially when considered with the values for LDL-cholesterol (also within the normal range in the study). This set of evidence suggests that the connection of the metabolic parameters of obese patients and coronary disease might not have a threshold below which the risk would be similar to the general healthy population.

The association between obesity and cerebrovascular disease, however, seems less evident. Large cohort studies have shown relationships ${ }^{31}$, but others were not able to confirm them ${ }^{41}$, in very distinct population, it is important to note. However, this disagreement may be due to the dichotomization of the classification of "healthy" and "unhealthy" obese 
individuals, since there is a clear correlation between cerebrovascular accident and adiposity when considering the parameters of systolic blood pressure $\geq 130$ $\mathrm{mmHg}$ and/or diastolic $\geq 85 \mathrm{mmHg}$, or hypertension treatment, fasting glucose $>100 \mathrm{mg} / \mathrm{dl}$, or diabetes mellitus treatment, and total cholesterol $>240 \mathrm{mg} /$ $\mathrm{dl}$ or dyslipidemia treatment ${ }^{41}$. The set of data indicates, thus, that the metabolic changes are necessary for the outcome of cerebrovascular disease to occur, unlike what happens with heart failure and, possibly, coronary artery disease.

\section{NEW AND OLD MOLECULES IN THE OBESITY AND ATHEROSCLEROSIS INTERFACE \\ iSGLT2, obesity, and atherosclerosis}

The iSGLT2 are a class of anti-diabetic drugs that inhibit the absorption of sodium and glucose in the proximal convoluted tubule, promoting osmotic diuresis and the renal glucose excretion ${ }^{42}$. Among the main effects of these drugs, are the reduction of blood glucose, arterial pressure (AP), and weight. All these effects can contribute to the reduction of atherosclerosis.

From the studies on safety requested by the American Food and Drug Administration (FDA) since 2009, interesting effects of anti-diabetic drugs have been observed in DM2 patients with high cardiovascular risk ${ }^{43}$. The use of empagliflozin in the Empla-REG study was associated with a $14 \%$ reduction (Risk ratio of 0.86; CI 95\%: 0.74-0.99; $p=0.04$ in the incidence of major cardiovascular events (Mace) after an average of 3.1 years, an effect induced, mostly, by the $38 \%$ reduction in mortality due to cardiovascular diseases (risk ratio of 0.62; CI 95\%: 0.49-0.77; $\mathrm{p}<0.001)$ and of $35 \%$ in admissions due to heart failure (risk ratio 0.65; CI 95\%: $0.50-0.85 ; \mathrm{p}=0.002)^{44}$. A similar result was observed in the Canvas study, using canagliflozin with a $14 \%$ reduction (risk ratio 0.86; CI 95\%: 0.75-0.97; $p=0.02$ ) of Mace incidence after 3.6 years $^{45}$. In uncontrolled studies conducted from databases, the so-called real-world studies, the lower incidence of events was observed with more intensity, with a $51 \%$ reduction of mortality due to all causes (risk ratio: 0.49; CI 95\%: 0.41-0.57; p < $0.001)^{46}$. The reasons behind this reduction remain unclear; however, it might be associated with the effects the iSGLT2 have on cardiovascular risk factors.

Gliflozins are associated with dose/response weight $\operatorname{loss}^{47}$, with an average reduction of $2.1 \mathrm{~kg}$ (CI
95\%: $-2.3--2.0 ; p<0.01)$ in comparison with the placebo after12 weeks of use ${ }^{48}$ and an average reduction of $2.9 \mathrm{~kg}$ (CI 95\%: $-3.72--2.07 ; \mathrm{p}<0.001)$ after two years, in comparison with other medications ${ }^{49}$. Still, the weight loss is predominantly of adipose tissue (in a $2: 1$ ratio $)^{50}$, particularly of visceral adipose tissue. The weight reduction is due, mostly, to glycosuria, with a estimated calorie deficit of $50 \mathrm{kcal} / \mathrm{day}^{51}$, despite the possible contribution of other mechanisms, such as the activation of brown adipose tissue and insulin reduction ${ }^{52}$.

The gliflozins promote a reduction of 3-5 $\mathrm{mmHg}$ in the systolic AP and 1-3 mmHg in the diastolic ${ }^{53}$. Mechanistically, the osmotic diuresis induced by the glycosuria contributes to the blood pressure reduction; however, other effects, such as the improvement of endothelial function ${ }^{54}$, arterial stiffness ${ }^{55}$, sympathetic tone ${ }^{56}$, and increase in natriuresis might be associated ${ }^{57}$.

Some studies have linked treatments with gliflozin to lower levels of inflammatory factors associated with atherosclerosis, such as the TNF-alpha ${ }^{58}$, interleukin 6 (IL-6), and monocyte chemoattractant protein-1 (MCP-1) 59 . In animal models, the treatment with iSGLT2 was capable of reducing the formation of atheromatous plaques in the aortic $\operatorname{arch}^{60}$, as well as the formation of foam cells in the atheromatous plaque $^{61}$. However, the iSGLT2 are associated with an increase in LDL, perhaps due to the reduction of the hepatic metabolism of these proteins ${ }^{62}$, and have a neutral effect on HDL. Still, no alterations were observed in the functions of HDL and the enzymes associated with this protein ${ }^{47,63}$. Studies on obese patients with no DM2 were not conducted. It is possible, however, that these effects are found mainly on DM2 patients, but not on those with regular blood glucose levels.

\section{Sphingolipids, lipid profile, and atherosclerosis}

The increase in FA levels results, also, from a reshaping of the synthesis of bioactive lipids, which might be involved in the physiopathology of conditions associated with obesity, such as diabetes and cardiovascular diseases. Among these bioactive lipids, the sphingolipids have been noted as an important structural membrane element and as molecules with cell signaling functions ${ }^{64}$. The sphingolipid de novo biosynthesis uses as a rate-limiting enzyme the serine palmitoyltransferase, whose catalytic activity has a significant increase with the increase of 
availability of palmitoyl-CoA, derived from a higher concentration of palmitate. Thus, a higher enzyme activity of the serine palmitoyltransferase results in a greater formation of several sphingolipids. Among them, the most involved in cellular processes are the ceramide and the sphingosine-1-phosphate, which implies directly in a larger pool fo these molecules in conditions of a higher supply of FAs. Such reshaping of sphingolipids can affect several tissues ${ }^{64,65}$.

Recently, plasma lipidomics data in the investigation of such bioactive lipids as biomarkers for unstable coronary artery disease in the retrospective cohort Lipid identified several lipid classes positively associated with future cardiovascular events, such as ceramides and sphingolipids, while lysophosphatidylcholine and diacylglycerol were negatively associated $^{66}$, which awakens an interest in other bioactive molecules as possible effectors of lipid signaling. In total, 53 lipid species were associated with cardiovascular events. Atherogenic lipoproteins, such as LDL and VLDL, are enriched in sphingolipids, which might also be associated with cardiovascular risk. However, the sphingolipids that are also associated with risk in the Lipid cohort had already been positively associated previously with unfavorable metabolic conditions, including dihydroceramide and different kinds of ceramides. These results suggest that the regulation might occur in a ceramide-synthase level, which has a distinct expression according to the tissue ${ }^{66}$.

Even though there are few divergent data, overall, the studies on lipidomic profile have shown a positive association between ceramides and several variables associated with metabolic disorders, such as atherosclerosis and cardiomyopathy due to lipotoxicity. Mechanisms of action of the ceramides intracellularly involve, classically, the induction of apoptosis, insulin resistance, endoplasmic reticulum stress, and opening of the permeability transition pore in the mitochondria. On the contrary, the inhibition of the biosynthesis of ceramides results in an improvement of several metabolic disorders in animal models, such as the decrease in the formation of atherosclerotic plaque $^{67}$.

The effector signaling of the sphingolipids sphingosine-1-phosphate deserves attention for its sophisticated and still not fully understood behavior (Figure 1). Initially, as the class of sphingolipids in general, during obesity in animal models, there is a positive regulation of the enzyme apparatus for the synthesis of ceramides with an increase of the sphingolipids and sphingosine-1-phosphate both in the plasma of obese mice as well as in adipocytes cultured in vitro. This increase culminates in the positive regulation of the cytokines involved in the inflammatory and pro-thrombotic status of obesity, possibly mediating atherosclerotic complications associated with weight gain $^{70}$. Such findings are corroborated by the positive association of the sphingosine-1-phosphate (S1P) with obesity in humans ${ }^{71}$ and by the experimental finding that it positively regulates the inflammatory pathway triggered by the TNF- $\alpha$ receptor, a vital element of the meta-inflammation triggered by obesity $^{72}$. Despite that, the S1P has a dual role ${ }^{73}$, since the signaling cascade triggered by the $\mathrm{S} 1 \mathrm{P}$ in the vascular endothelium and in the cardiomyocyte has a protective role, regulating the vascular development, migration, angiogenesis, vasodilation signaling, and microvascular barrier function, especially when the S1P é chaperoned by the HDL or the apolipoprotein $\mathrm{M}^{74,75}$, a condition in which the HDL/S1P induces a complex with the $\beta$-arrestin in order to suppress the NF-KB activation induced by cytokines. Besides, the signaling of the S1P1 receptor is vessel-protective, since its EC-specific super expression protects from the formation of plaques in atherosclerosis models in a diet rich in fat ${ }^{75}$. Unfortunately, there is still a gap concerning the specific reshaping of these sphingolipids in obesity and its role in the vascular endothelium during obesity, although some light has been shed on the protective role of the S1P1 and one of its ligands (S1P carried by the HDL or ApoM). However, it is also reasonable to extend this atherogenic environment to the changes in the content of the dysfunctional HDL S1P of both diabetic and coronary artery disease patients ${ }^{76,77}$, who have a decreased capacity to carry S1P. Indeed, since the biosynthesis pathway of sphingolipids is interconnected, the therapeutic modulation of any element would be complicated, and so far unpredictable, and the ceramide-synthase would have a pathogenic role in a given context ${ }^{64}$, or even on how the entire system would behave in the endothelium of an obese individual.

Unsaturated fatty acids, diet, and atherosclerosis

Lipids have been widely studied in the context of cardiovascular diseases, and the dietary recommendations for the ingestion of the macronutrients have emphasized the replacement of saturated FAs 


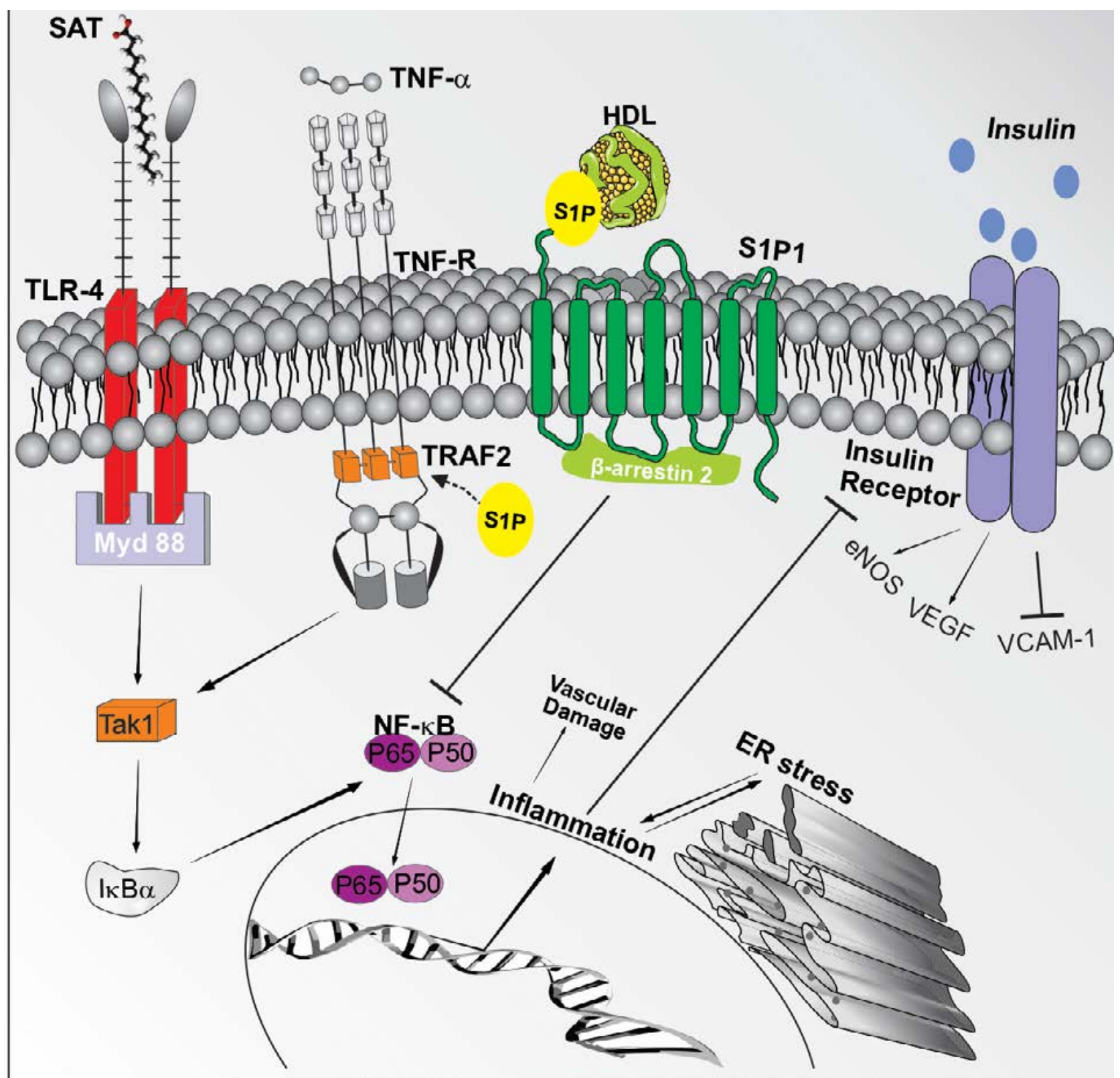

FIGURE 1 (adapted from Moura-Assis et al. ${ }^{68}$ ). Activation of inflammatory signaling by saturated fatty acids in conditions associated with obesity and their interaction with the S1P The TAK1 (Transforming growth factor b-activated kinase 1) is activated by several stimuli, including cytokines, TLRs, and factors associated with the TNF receptor (Traf), which culminates in the activation of the central pathway IkB kinase (IKK)-nuclear factor-kB (NF-kB), responsible for the activation of transcription of several inflammatory genes ${ }^{69}$ and the blocking of the insulin receptor. This scheme also demonstrates that the S1Pmolecule, when generated intracellularly, acts as a secondary lipidic messenger, connecting itself to the Traf2 and promoting changes in the complex with Traf2 that are crucial to the inflammatory activation of the IKK- NF-kB ${ }^{70}$. When S1P is carried by HDL or ApoM, when it connects to the endothelial cell, it promotes the formation of a S1P1- $\beta$-arrestin 2 complex that inhibits the activation of $N F-k B$ and blocks vascular inflammation.

for unsaturated FAs since the 1960s. The debates around the recommendations for the ingestion of FAs occupy a prominent position on the agenda of most dietary guides and, according to the American guide, the ingestion of saturated FAs should no exceed the daily limit of $10 \%$ of the total energetic value. The dietary guidelines for the Brazilian population, in turn, was structured to stimulate dietary patterns and healthy behavior to the detriment of recommen- dations of individual nutrients. Such action is necessary since nutrients per se seem to not represent a sine qua non-condition for the development of diseases. However, even within the perspective of the Brazilian dietary guide, there is an instruction towards the reduction of saturated fats, substituting them for unsaturated ones.

Even though there are multiple associations between lipid consumption and the development of 
cardiovascular diseases, so far, no evidence has been established through well-controlled clinical trials. Besides, the massive divergence between dietary patterns between countries makes it substantially more challenging to create guidelines.

Some studies designed to assess the effect of the replacement of saturated FAs by carbohydrates indicate there are no overall differences in the markers for cardiovascular risk prediction such as LDL-c e HDL-c ${ }^{78}$. However, some studies that emphasized the replacement of saturated FAs by carbohydrates with low glycemic indexes found a reduction of LDL-c and increase in HDL-c ${ }^{79}$. These opposite effects emphasize that specifying the source of foods that make up the replacement list is mandatory and the use of carbohydrates with low glycemic index as replacements for saturated FAs confers cardiovascular benefits. A recent analysis of the Nurses' Health Study (NHS) and the Health Professionals Follow-up Study (HPFS) estimated that the isocaloric replacement of $5 \%$ of the saturated FAs by polyunsaturated, monounsaturated Fas, and carbohydrates with low glycemic index represented a reduction of $25 \%, 15 \%$, and $9 \%$ of cardiovascular risk, respectively ${ }^{80}$.

Since the epidemiological findings on the low incidence of cardiovascular mortality among the Inuit of Greenland, the consumption of fish and the omega-3 FAs have been extensively studied, In general, prospective studies suggest a lower risk of coronary disease in individuals with no previous cardiovascular disease who have a higher intake of polyunsaturated or fish $\mathrm{FAs}^{81}$. The randomized clinical trials who did find benefits in the supplementation with fish oil in individuals with cardiovascular disease present certain limitations and need to be interpreted with caution. Individuals with cardiovascular disease already receive, in general, pharmacological treatment (statins, for example), and the supplementation with fish oil hardly potentializes the action of these drugs. In addition, the benefits of the omega- 3 FAs seem to have a threshold, and additional doses do not confer an increment to the cardiovascular protection in individuals who already have an adequate intake of these FAs in their diet.

Some mechanistic studies in rodents have dissected with greater precision the effects of different types of FAs in the metabolism and in their effect as signaling molecules. Polyunsaturated FAs can increase the expression of genes involved in the oxidation of lipids (PPAR $\alpha)$ and decrease the expression of those involved in the hepatic lipogenesis (SREBP-1c), decreasing atherosclerotic injuries ${ }^{82,83}$. Additionally, the partial and isocaloric replacement of saturated FAs by unsaturated FAs decreases inflammation and the endoplasmic reticulum stress in the aorta of obese and insulin-resistant mice ${ }^{68}$. On the other hand, saturated FAs have been associated with an increase of endothelial inflammation and greater atherosclerotic injury in diet-induced obese mice ${ }^{82}$. Such effects seem to be related with the activation of the pattern recognition toll-like receptor 4 (TLR4) of the innate immune system and its inflammatory cascade $^{84}$.

Beyond the consensual difficulties, adherence to the traditional Mediterranean diet has been associated with lower cardiovascular risk and is widely encouraged for the high-risk population. Monounsaturated FAs, especially the oleic acid, represent between $16-29 \%$ of the total energetic value ${ }^{85}$ and their inclusion in diet, as a replacement for simple carbohydrates, is significantly associated with a reduction in mortality ${ }^{81}$. Additionally, the Predimed study (Prevención con Dieta Mediterránea) found a reduction in cardiovascular events in groups that received supplementation with extra-virgin olive oil or oilseeds in comparison with the control group in a five-year follow-up with men and women with no cardiovascular disease but high-risk. The individuals placed in the supplement group were instructed to consume at least 50 grams of olive oil or 30 grams of oilseeds, including nuts, almonds, and hazelnuts ${ }^{86}$. Finally, the impact of this diet on effector mechanisms for the protection of the activated endothelial cells in obese individuals is still not clear.

\section{CONCLUSIONS}

The increase in the overall prevalence of obesity is associated with an increase in cardiovascular risk. There is piling evidence that demonstrates that obesity promotes macrostructural cardiovascular changes and bad cellular metabolic adjustments in the vascular endothelium, a vital element of the onset of the atherosclerotic process. Endothelial activation is triggered, initially, by the plasma content of free fatty acids in obese individuals, but also promotes a series of metabolic adjustments in several tissues from the reshaping of the bioactive lipid pool, which are capable of controlling other pathways of cellular and protective stress. The scientific advancement in this 
is due to the incorporation of several tools that allow for the study of lipids at a omic perspective, as well for the integration of such knowledge to a knowledge of cell signaling and population data, which has allowed for the progress concerning the identification of new biomarkers and new therapeutic targets. Indeed, over the next years, there will probably be more pieces available to this network of interdisciplinary knowledge that goes beyond the from bench to bedside limitations.

\section{RESUMO}

A aterosclerose é a causa líder de mortalidade no mundo contemporâneo. O papel central da célula endotelial (EC) na homeostase vascular, as alterações metabólicas que ocorrem quando a célula se torna ativada e os elementos envolvidos nesses processos vêm sendo bastante explorados nos últimos anos. A obesidade e o seu impacto, promovendo uma elevação dos níveis sanguíneos de ácidos graxos (FAs) livres, é bastante associada à aterosclerose e à mortalidade cardiovascular. Entretanto, os mecanismos que promovem alterações estruturais cardiovasculares e alterações adaptativas nas ECs, particularmente no contexto da obesidade, são pouco conhecidos. Aqui, nós revisamos estudos que avaliaram as adaptações metabólicas das ECs normais e disfuncionais durante exposição a FAs, bem como as perspectivas epidemiológicas das alterações cardiovasculares estruturais na obesidade. Finalmente, exploramos o papel de novos atores - esfingolípides, ácidos graxos insaturados da dieta e inibidores do cotransportador de sódio-glucose 2 (iSGLT2) - na aterosclerose e sua relação com a obesidade.

PALAVRAS-CHAVE: Obesidade. Fatores de risco. Aterosclerose. Endotélio.

\section{REFERENCES}

1. Pircher A, Treps L, Bodrug N, Carmeliet P. Endothelial cell metabolism: a novel player in atherosclerosis? Basic principles and therapeutic opportunities. Atherosclerosis. 2016;253:247-57.

2. Gimbrone Jr MA, García-Cardeña G. Endothelial cell dysfunction and the pathobiology of atherosclerosis. Circ Res. 2016;118(4):620-36.

3. NCD Risk Factor Collaboration (NCD-RisC). Worldwide trends in bodymass index, underweight, overweight, and obesity from 1975 to 2016: a pooled analysis of 2416 population-based measurement studies in 128.9 million children, adolescents, and adults. Lancet. 2017;390(10113):2627-42.

4. Neeland IJ, Poirier P, Després JP. Cardiovascular and metabolic heterogeneity of obesity: clinical challenges and implications for management. Circulation. 2018;137(13):1391-406.

5. Kim SH, Després JP, Koh KK. Obesity and cardiovascular disease: friend or foe? Eur Heart J. 2016;37(48):3560-8.

6. Neeland IJ, Turer AT, Ayers CR, Berry JD, Rohatgi A, Das SR, et al. Body fat distribution and incident cardiovascular disease in obese adults. . Am Coll Cardiol. 2015;65(19):2150-1.

7. Després JP. Body fat distribution and risk of cardiovascular disease: an update. Circulation. 2012;126(10):1301-13.

8. Cepeda-Valery B, Pressman GS, Figueredo VM, Romero-Corral A. Impact of obesity on total and cardiovascular mortality: fat or fiction? Nat Rev Cardiol. 2011;8(4):233-7.

9. Rask-Madsen C, King GL. Vascular complications of diabetes: mechanisms of injury and protective factors. Cell Metab. 2013;17(1):20-33.

10. King GL, Park K, Li Q. Selective insulin resistance and the development of cardiovascular diseases in diabetes: the 2015 Edwin Bierman Award Lecture. Diabetes. 2016;65(6):1462-71.

11. Bierhans L, Conradi LC, Treps L, Dewerchin M, Carmeliet P. Central role of metabolism in endothelial cell function and vascular disease. Physiology (Bethesda). 2017;32(2):126-40.

12. Eelen $G$, de Zeeuw $P$, Simons $M$, Carmeliet P. Endothelial cell metabolism in normal and diseased vasculature. Circ Res. 2015;116(7):1231-44.

13. Groschner LN, Waldeck-Weiermair M, Malli R, Graier WF. Endothelial mitochondria: less respiration, more integration. Pflugers Arch. 2012;464(1):63-76.

14. Lee DC, Sohn HA, Park ZY, Oh S, Kang YK, Lee KM, et al. A lactate-induced response to hypoxia. Cell. 2015;161(3):595-609.

15. Krützfeldt A, Spahr R, Mertens S, Siegmund B, Piper HM. Metabolism of exogenous substrates by coronary endothelial cells in culture. J Mol Cell Cardiol. 1990;22(12):1393-404.

16. Dagher Z, Ruderman N, Tornheim $K$, Ido Y. Acute regulation of fatty acid oxidation and amp-activated protein kinase in human umbilical vein endothelial cells. Circ Res. 2001;88(12):1276-82.

17. Bastin J. Regulation of mitochondrial fatty acid $\beta$-oxidation in human: what can we learn from inborn fatty acid $\beta$-oxidation deficiencies? Biochimie. 2014;96:113-20.

18. Eelen G, Zeeuw P, Treps L, Harjes U, Wong BW, Carmeliet P. Endothelial cell metabolism. Physiol Rev. 2018;98(1):3-58.

19. Schoors S, Bruning U, Missiaen R, Queiroz KC, Borgers G, Elia I, et al. Fatty acid carbon is essential for dNTP synthesis in endothelial cells. Nature. 2015;520(7542):192-7.

20. Goldberg IJ, Bornfeldt KE. Lipids and the endothelium: bidirectional interactions. Curr Atheroscler Rep. 2013;15(11):365.

21. Wilhelm K, Happel K, Eelen G, Schoors S, Oellerich MF, Lim R, et al. FOXO1 couples metabolic activity and growth state in the vascular endothelium. Nature. 2016;529(7585):216-20.

22. Kalucka J, BierhansI L, Conchinha NV, Missiaen R, Elia I, Brüning U, et al. Quiescent endothelial cells upregulate fatty acid $\beta$-oxidation for vasculoprotection via redox homeostasis. Cell Metab. 2018;S1550-4131(18)304595.

23. Steinberg HO, Tarshoby M, Monestel R, Hook G, Cronin J, Johnson A, et al. Elevated circulating free fatty acid levels impair endothelium-dependent vasodilation. J Clin Invest. 1997;100(5):1230-9.

24. Theodorou K, Boon RA. Endothelial cell metabolism in atherosclerosis. Front Cell Dev Biol. 2018;6:82.

25. Steinberg $\mathrm{HO}$, Chaker $\mathrm{H}$, Leaming R, Johnson A, Brechtel G, Baron AD. Obesity/insulin resistance is associated with endothelial dysfunction. Implications for the syndrome of insulin resistance. J Clin Invest. 1996;97(11):2601-10.

26. Inoguchi T, Li P, Umeda F, Yu HY, Kakimoto M, Imamura M, et al. High glucose level and free fatty acid stimulate reactive oxygen species production through protein kinase $C$ : dependent activation of $N A D(P) H$ oxidase in cultured vascular cells. Diabetes. 2000;49(11):1939-45.

27. Li X, Gonzalez O, Shen X, Barnhart S, Kramer F, Kanter JE, et al. Endothelial acyl-CoA synthetase 1 is not required for inflammatory and apoptotic effects of a saturated fatty acid-rich environment. Arterioscler Thromb Vasc Biol. 2013;33(2):232-40.

28. Won JC, Park JY, Kim YM, Koh EH, Seol S, Jeon BH, et al. Peroxisome proliferator-activated receptor-gamma coactivator 1-alpha overexpression prevents endothelial apoptosis by increasing ATP/ADP translocase activity. Arterioscler Thromb Vasc Biol. 2010;30(2):290-7.

29. Stolberg CR, Mundbjerg LH, Funch-Jensen P, Gram B, Bladbjerg EM, Juhl CB. Effects of gastric bypass surgery followed by supervised physical train- 
ing on inflammation and endothelial function: a randomized controlled trial. Atherosclerosis. 2018;273:37-44.

30. Grenier-Larouche T, Carreau AM, Geloën A, Frisch F, Biertho L, Marceau $S$, et al. Fatty acid metabolic remodeling during type 2 diabetes remission after bariatric surgery. Diabetes. 2017;66(11):2743-55.

31. Caleyachetty R, Thomas GN, Toulis KA, Mohammed N, Gokhale KM, Balachandran K, et al. Metabolically healthy obese and incident cardiovascular disease events among 3.5 million men and women. | Am Coll Cardiol. 2017;70(12):1429-37.

32. Zheng R, Zhou D, Zhu Y. The long-term prognosis of cardiovascular disease and all-cause mortality for metabolically healthy obesity: a systematic review and meta-analysis. I Epidemiol Community Health. 2016;70(10):1024-31.

33. Kramer CK, Zinman B, Retnakaran R. Are metabolically healthy overweight and obesity benign conditions? A systematic review and meta-analysis. Ann Intern Med. 2013;159(11):758-69.

34. Fan J, Song Y, Chen Y, Hui R, Zhang W. Combined effect of obesity and cardio-metabolic abnormality on the risk of cardiovascular disease: a meta-analysis of prospective cohort studies. Int J Cardiol. 2013;168(5):4761-8.

35. Cil H, Bulur S, Turker Y, Kaya A, Alemdar R, Karabacak A, et al. Impact of body mass index on left ventricular diastolic dysfunction. Echocardiography. 2012;29(6):647-51.

36. Ng ACT, Prevedello F, Dolci G, Roos C], Djaberi R, Bertini M, et al. Impact of diabetes and increasing body mass index category on left ventricular systolic and diastolic function. J Am Soc Echocardiogr. 2018;31(8):916-25.

37. Cheng S, Xanthakis V, Sullivan LM, Lieb W, Massaro J, Aragam J, et al. Correlates of echocardiographic indices of cardiac remodeling over the adult life course: longitudinal observations from the Framingham Heart Study. Circulation. 2010;122(6):570-8.

38. Blomstrand P, Sjoblom P, Nilsson M, Wijkman M, Engvall M, Länne T, et al. Overweight and obesity impair left ventricular systolic function as measured by left ventricular ejection fraction and global longitudinal strain. Cardiovasc Diabetol. 2018;17:113

39. Berg TJ, Snorgaard O, Faber J, Torjesen PA, Hildebrandt P, Mehlsen J, et al. Serum levels of advanced glycation end products are associated with left ventricular diastolic function in patients with type 1 diabetes. Diabetes Care. 1999;22(7):1186-90

40. Chang Y, Kim BK, Yun KE, Cho J, Zhang Y, Rampal S, et al. Metabolically-healthy obesity and coronary artery calcification. I Am Coll Cardiol. 2014;63(24):2679-86.

41. Lee HJ, Choi EK, Lee SH, Kim YJ, Han KD, Oh S. Risk of ischemic stroke in metabolically healthy obesity: a nationwide population-based study. PLoS One. 2018:13(3):e0195210.

42. Zelniker TA, Braunwald E. Cardiac and renal effects of sodium-glucose co-transporter 2 inhibitors in diabetes: |ACC state-of-the-art review. | Am Coll Cardiol. 2018;72(15):1845-55.

43. Lim S, Eckel RH, Koh KK. Clinical implications of current cardiovascular outcome trials with sodium glucose cotransporter-2 (SGLT2) inhibitors. Atherosclerosis. 2018;272:33-40.

44. Zinman B, Wanner C, Lachin IM, Fitchett D, Bluhmki E, Hantel S, et al. Empagliflozin, cardiovascular outcomes, and mortality in type 2 diabetes. N Engl | Med. 2015;373(22):2117-28.

45. Neal B, Perkovic V, Matthews DR. Canagliflozin and cardiovascular and renal events in type 2 diabetes. N Engl | Med. 2017;377(21):2099.

46. Kosiborod M, Cavender MA, Fu AZ, Wilding IP, Khunti K, Holl RW, et al. Lower risk of heart failure and death in patients initiated on sodium-glucose cotransporter-2 inhibitors versus other glucose-lowering drugs: the CVD-REAL Study (Comparative effectiveness of cardiovascular outcomes in new users of sodium-glucose cotransporter-2 inhibitors). Circulation. 2017;136(3):249-59.

47. Fadini GP, Bonora BM, Mayur S, Rigato M, Avogaro A. Dipeptidyl peptidase-4 inhibitors moderate the risk of genitourinary tract infections associated with sodium-glucose co-transporter-2 inhibitors. Diabetes Obes Metab. 2018;20(3):740-4

48. Storgaard H, Gluud LL, Bennett C, Grøndahl MF, Christensen MB, Knop $\mathrm{FK}$, et al. Benefits and harms of sodium-glucose co-transporter 2 inhibitors in patients with type 2 diabetes: a systematic review and meta-analysis. PLoS One. 2016;11(11):e0166125.

49. Zhang XL, Zhu QQ, Chen YH, Li XL, Chen F, Huang JA, et al. Cardiovascular safety, long-term noncardiovascular safety, and efficacy of sodium-glucose cotransporter 2 inhibitors in patients with type 2 diabetes mellitus: a systemic review and meta-analysis with trial sequential analysis. J Am Heart Assoc. 2018;7(2):pii: e007165.

50. Bolinder J, Ljunggren Ö, Kullberg J, Johansson L, Wilding J, Langkilde AM et al. Effects of dapagliflozin on body weight, total fat mass, and regional adipose tissue distribution in patients with type 2 diabetes mellitus with inadequate glycemic control on metformin. J Clin Endocrinol Metab. 2012;97(3):1020-31.

51. Ferrannini $G$, Hach $T$, Crowe $S$, Sanghvi $A$, Hall KD, Ferrannini E. Energy balance after sodium-glucose cotransporter 2 inhibition. Diabetes Care. 2015;38(9):1730-5.

52. Xu L, Nagata N, Nagashimada M, Zhuge F, Ni Y, Chen G, et al. SGLT2 Inhibition by empagliflozin promotes fat utilization and browning and attenuates inflammation and insulin resistance by polarizing $\mathrm{m} 2$ macrophages in diet-induced obese mice. EBioMedicine. 2017;20:137-49.

53. Sarafidis PA, Lazaridis AA, Ruiz-Hurtado G, Ruilope LM. Blood pressure reduction in diabetes: lessons from ACCORD, SPRINT and EMPA-REG OUTCOME. Nat Rev Endocrinol. 2017:13(6):365-74.

54. Shigiyama F, Kumashiro N, Miyagi M, Ikehara K, Kanda E, Uchino H, et al. Effectiveness of dapagliflozin on vascular endothelial function and glycemic control in patients with early-stage type 2 diabetes mellitus: DEFENCE study. Cardiovasc Diabetol. 2017;16(1):84

55. Pfeifer M, Townsend RR, Davies MI, Vijapurkar U, Ren |. Effects of canagliflozin, a sodium glucose co-transporter 2 inhibitor, on blood pressure and markers of arterial stiffness in patients with type 2 diabetes mellitus: a post hoc analysis. Cardiovasc Diabetol. 2017;16(1):29.

56. Wan N, Rahman A, Hitomi H, Nishiyama A. The effects of sodium-glucose cotransporter 2 inhibitors on sympathetic nervous activity. Front Endocrinol (Lausanne). 2018;9:421.

57. León Jiménez D, Cherney DZI, Bjornstad P, Castilla Guerra L, Miramontes González IP. Antihyperglycemic agents as novel natriuretic therapies in diabetic kidney disease. Am J Physiol Renal Physiol. 2018. doi: 10.1152/ ajprenal.00384.2017.

58. Birnbaum Y, Bajaj M, Yang HC, Ye Y. Combined SGLT2 and DPP4 inhibition reduces the activation of the Nlrp3/asc inflammasome and attenuates the development of diabetic nephropathy in mice with type 2 diabetes. Cardiovasc Drugs Ther. 2018;32(2):135-45.

59. Mancini S), Boyd D, Katwan OJ, Strembitska A, Almabrouk TA, Kennedy S, et al. Canagliflozin inhibits interleukin-1 $\beta$-stimulated cytokine and chemokine secretion in vascular endothelial cells by AMP-activated protein kinase-dependent and -independent mechanisms. Sci Rep. 2018;8(1):5276

60. Nasiri-Ansari N, Dimitriadis GK, Agrogiannis G, Perrea D, Kostakis ID, Kaltsas $\mathrm{G}$, et al. Canagliflozin attenuates the progression of atherosclerosis and inflammation process in APOE knockout mice. Cardiovasc Diabetol. 2018;17(1):106.

61. Terasaki M, Hiromura M, Mori Y, Kohashi K, Nagashima M, Kushima H, et al. Amelioration of hyperglycemia with a sodium-glucose cotransporter 2 inhibitor prevents macrophage-driven atherosclerosis through macrophage foam cell formation suppression in type 1 and type 2 diabetic mice. PLoS One. 2015;10(11):e0143396.

62. Briand F, Mayoux E, Brousseau E, Burr N, Urbain I, Costard C, et al. Empagliflozin, via switching metabolism toward lipid utilization, moderately increases LDL cholesterol levels through reduced LDL catabolism. Diabetes. 2016;65(7):2032-8.

63. Fadini GP, Bonora BM, Zatti G, Vitturi N, lori E, Marescotti MC, et al. Effects of the SGLT2 inhibitor dapagliflozin on HDL cholesterol, particle size, and cholesterol efflux capacity in patients with type 2 diabetes: a randomized placebo-controlled trial. Cardiovasc Diabetol. 2017;16(1):42.

64. Cowart LA. Sphingolipids: players in the pathology of metabolic disease. Trends Endocrinol Metab. 2009;20(1):34-42

65. Choi S, Snider AJ. Sphingolipids in high fat diet and obesity-related diseases. Mediators Inflamm. 2015;2015:520618.

66. Mundra PA, Barlow CK, Nestel PJ, Barnes EH, Kirby A, Thompson P, et al. Large-scale plasma lipidomic profiling identifies lipids that predict cardiovascular events in secondary prevention. JCI Insight. 2018;3(17).

67. Chaurasia B, Summers SA. Ceramides: lipotoxic inducers of metabolic disorders. Trends Endocrinol Metab. 2015;26(10):538-50.

68. Moura-Assis A, Afonso MS, Oliveira V, Morari J, Santos GA, Koike M, et al. Flaxseed oil rich in omega-3 protects aorta against inflammation and endoplasmic reticulum stress partially mediated by GPR120 receptor in obese, diabetic and dyslipidemic mice models. J Nutr Biochem. 2018;53:9-19.

69. Ajibade AA, Wang HY, Wang RF. Cell type-specific function of TAK1 in 
innate immune signaling. Trends Immunol. 2013;34(7):307-16.

70. Samad F, Hester KD, Yang G, Hannun YA, Bielawski J. Altered adipose and plasma sphingolipid metabolism in obesity: a potential mechanism for cardiovascular and metabolic risk. Diabetes. 2006;55(9):2579-87.

71. Kowalski GM, Carey AL, Selathurai A, Kingwell BA, Bruce CR. Plasma sphingosine-1-phosphate is elevated in obesity. PLoS One. 2013;8(9):e72449.

72. Alvarez SE, Harikumar KB, Hait NC, Allegood I, Strub GM, Kim EY, et al. Sphingosine-1-phosphate is a missing cofactor for the E3 ubiquitin ligase TRAF2. Nature. 2010;465(7301):1084-8.

73. Lee JF, Zeng Q, Ozaki H, Wang L, Hand AR, Hla T, et al. Dual roles of tight junction-associated protein, zonula occludens-1, in sphingosine 1-phosphate-mediated endothelial chemotaxis and barrier integrity. I Biol Chem. 2006;281(39):29190-200.

74. Proia RL, Hla T. Emerging biology of sphingosine-1-phosphate: its role in pathogenesis and therapy. I Clin Invest. 2015;125(4):1379-87.

75. Galvani S, Sanson M, Blaho VA, Swendeman SL, Obinata H, Conger H, et al. HDL-bound sphingosine 1-phosphate acts as a biased agonist for the endothelial cell receptor S1P1 to limit vascular inflammation. Sci Signal. 2015;8(389):ra79.

76. Keul P, Polzin A, Kaiser K, Gräler M, Dannenberg L, Daum G, et al. Potent anti-inflammatory properties of HDL in vascular smooth muscle cells mediated by HDL-S1P and their impairment in coronary artery disease due to lower HDL-S1P: a new aspect of HDL dysfunction and its therapy. FASEB J. 2018:fj201801245R.

77. Frej $C$, Mendez Al, Ruiz $M$, Castillo $M$, Hughes TA, Dahlbäck $B$, et al. A shift in ApoM/STP between HDL-particles in women with type 1 diabetes mellitus is associated with impaired anti-inflammatory effects of the
ApoM/S1P complex. Arterioscler Thromb Vasc Biol. 2017;37(6):1194-205.

78. Mensink RP, Zock PL, Kester AD, Katan MB. Effects of dietary fatty acids and carbohydrates on the ratio of serum total to HDL cholesterol and serum lipids and apolipoproteins: a meta-analysis of 60 controlled trials. Am | Clin Nutr. 2003;77(5):1146-55.

79. Levitan EB, Cook NR, Stampfer MJ, Ridker PM, Rexrode KM, Buring JE, et al. Dietary glycemic index, dietary glycemic load, blood lipids, and C-reactive protein. Metabolism. 2008;57(3):437-43.

80. Li Y, Hruby A, Bernstein AM, Ley SH, Wang DD, Chiuve SE, et al. Saturated fats compared with unsaturated fats and sources of carbohydrates concerning risk of coronary heart disease: a prospective cohort study. I Am Coll Cardiol. 2015;66(14):1538-48.

81. Wang DD, Li Y, Chiuve SE, Stampfer MJ, Manson JE, Rimm EB, et al. Association of specific dietary fats with total and cause-specific mortality. IAMA Intern Med. 2016;176(8):1134-45.

82. Lottenberg AM, Afonso MS, Lavrador MS, Machado RM, Nakandakare ER. The role of dietary fatty acids in the pathology of metabolic syndrome. J Nutr Biochem. 2012;23(9):1027-40.

83. Afonso MS, Castilho G, Lavrador MS, Passarelli M, Nakandakare ER, Lottenberg SA, et al. The impact of dietary fatty acids on macrophage cholesterol homeostasis. J Nutr Biochem. 2014;25(2):95-103.

84. Velloso LA, Folli F, Saad MI. TLR4 at the crossroads of nutrients, gut microbiota, and metabolic inflammation. Endocr Rev. 2015;36(3):245-71.

85. Wang DD, Hu FB. Dietary fat and risk of cardiovascular disease: recent controversies and advances. Annu Rev Nutr. 2017;37:423-46.

86. Estruch R, Ros E, Salas-Salvadó J, Covas MI, Corella D, Arós F, et al. Primary prevention of cardiovascular disease with a mediterranean diet supplemented with extra-virgin olive oil or nuts. N Engl | Med. 2018;378(25):e34 